\title{
Optimized Energy Management of Inductively Charged Electric Buses Reflecting Operational Constraints and Traffic Conditions
}

\author{
Tamás Kurzevezil \\ Technische Universität Braunschweig \\ Institut für Verkehrssicherheit und \\ Automatisierungstechnik \\ Braunschweig, Germany \\ kurczveil@iva.ing.tu-bs.de
}

\author{
Lars Schnieder, Felix Burmeister \\ German Aerospace Center \\ Institute of Transportation Systems \\ Braunschweig, Germany \\ lars.schnieder@dlr.de \\ felix.burmeister@dlr.de
}

\begin{abstract}
The introduction of alternative propulsion concepts in public transport makes significant contributions to further reduce pollutants emitted by transport systems (noise, air pollutants). This paradigm shift is a major challenge for public transport operators. Operational performance of heavy duty vehicles depends on many different factors such as climatic conditions, load profiles (e.g. variations in passenger occupancy) and track topology. Currently there is no common standard which allows the assessment of energetic performance of different vehicle concepts and optimum deployment strategies of electric charging infrastructure in the network (with respect to their impact on the vehicles' duty plans). This paper introduces a simulation-based approach to provide answers to this multivariant optimization problem. The model outlined in this paper allows operators to choose the most adequate vehicle type(s) and corresponding infrastructure for their respective conditions aiming at a maximum operational availability of the buses during the operational day. Furthermore, the simulation model allows the assessment of time tables and line topologies with respect to their operational feasibility. This paper discusses experiences made with simulative studies in the introduction of inductively charged electric buses in the city of Braunschweig (Germany).
\end{abstract}

Keywords- Inductive charging, public transport, traffic flow simulation

\section{INTRODUCTION}

The introduction of new and innovative vehicle concepts in public transport requires improvements in the economic efficiency and reliability of transport operations. With the usage of electrical vehicles, there are restrictions that need to be considered in addition to the already existing problems of additional vehicle scheduling of public transport companies [1]. Firstly, the vehicles have a much smaller range due to battery capacity or fuel restriction than traditional diesel vehicles and secondly they can be recharged or refuelled at specific stations only. In addition to this, the interdependency between the dimensioning of power electronics and drive aggregates of the vehicle on the one side and the location and number of charging infrastructure on the other side need to be taken into consideration in order to define an economically feasible migration scenario.

\section{A. Operation of electrical busses as a complex planning problem}

Due to the long duration of use of assets in public transport, correct positioning and dimensioning of infrastructure is essential. It must be determined, whether the charging infrastructure to be deployed is located at specific points (e.g. stops), across segments (e.g. bus-lanes) of if a battery with low energy can be replaced with another one [2]. Furthermore, life-cycle costs of stationary infrastructure and compatible vehicles as well as the interrelation between these two aspects need to be taken into account. Currently, traction batteries significantly determine the investment as well as vehicle weights. These factors have to be weighed up against the required high level of operational availability.

\section{B. The goal: Economic efficiency and availability of vehicles on line-service}

When introducing electrically charged busses, the planning process aims for the maximization of operational availability (increased up-time, reduced down-time). The increase of uptime can be achieved through the energetic optimization of drive-cycles and traffic flow as well as the improvement of energetic performance of the vehicle's auxiliaries. Due to the limited capacity of available energy storage systems, current electrical vehicles will require repeated recharges throughout the day, which represent a main factor of down-time in their public transport application. Measures for reducing this downtime aim for synchronizing charging times with vehicles' standstill periods (e.g. traffic signals, regular stops, rest periods of drivers) and charging locations (e.g. where high colocation of compatible vehicles is expected). 
2015 Models and Technologies for Intelligent Transportation Systems (MT-ITS)

3-5. June 2015. Budapest, Hungary

\section{Experiences from current projects}

Funded by the German Federal Ministry of Transport, Building and Urban Development, the aim of the current project emil (Elektromobilität mittels induktiver Ladung electric mobility via inductive charging) is to integrate an inductive vehicle charging system and a compatible prototype bus fleet into Braunschweig's traffic and infrastructure. The bus lines that are involved are the M29 and M19 that circle the city centre counter-clockwise and clockwise, respectively. They carry the highest percentage of Braunschweig's publicly transported passengers with a high service frequency from and to major traffic nodes and landmarks (e.g. main station, university). So far, five busses were introduced into regular service that are being charged at three positions (one at a regular bus stops, one at the final stop and one at the bus depot). A fourth charging system is to be installed at another bus stop. With inductive charging, the charging-process can be initiated automatically without a physical connection to the infrastructure. It therefore decreases the time needed for charging, relieves the driver of any necessary actions and does not obstruct passenger service [3].

\section{Simulation-BASED PlanNing OF Electrical BuS OPERATIONS}

In order to evaluate the multi-variant optimization problem, an approach based on a traffic-flow simulation has been chosen. Due to the fact that currently available microscopic traffic flow simulation software tools mostly only offer rudimentary solutions for recreating the energy content of vehicle objects and its parameter- and trajectory dependent variation (consumption), a consumption model for the energetic state of vehicles has been developed. This model consists of two complementary parts (see Figure 1 for the holistic approach of energetic optimization of electrical bus operations): A sub-model for energy consumption (section A) and a sub-model of vehicle charging (section B). With these models in place, the evaluation of the application of novel energy-supply and drive concepts can be achieved. The developed simulation tool allows the instantiation of traffic scenarios based on measured field data from public transport vehicles, including vehicle position and drivetrain measurements, along with operational characteristics. The microscopic-traffic simulation tool SUMO (Simulation of Urban Mobility) of the German Aerospace Centre (DLR) is being applied. As depicted in Figure 1, the vehicles integration into intelligent transportation systems (ITS) has to be reflected. ITS are advanced applications which aim to provide innovative services relating to different transportation modes and traffic management with the primary goal of supplying users and underlying functions with required information to make safer, more coordinated and 'smarter' use of resources. With the application of ITS, public transport vehicles could optimize their decisions and adapt their trajectory towards an energy optimal one [4]. As shown in figure 1 the vehicles' integration into electricity grid has to be considered as well. It becomes clear that electric buses have to gather and act on information, such as about the behaviour of energy sources and other consumers as well as provide information required by other participants to improve the efficiency, reliability, economics, and sustainability of the production and distribution of electricity.

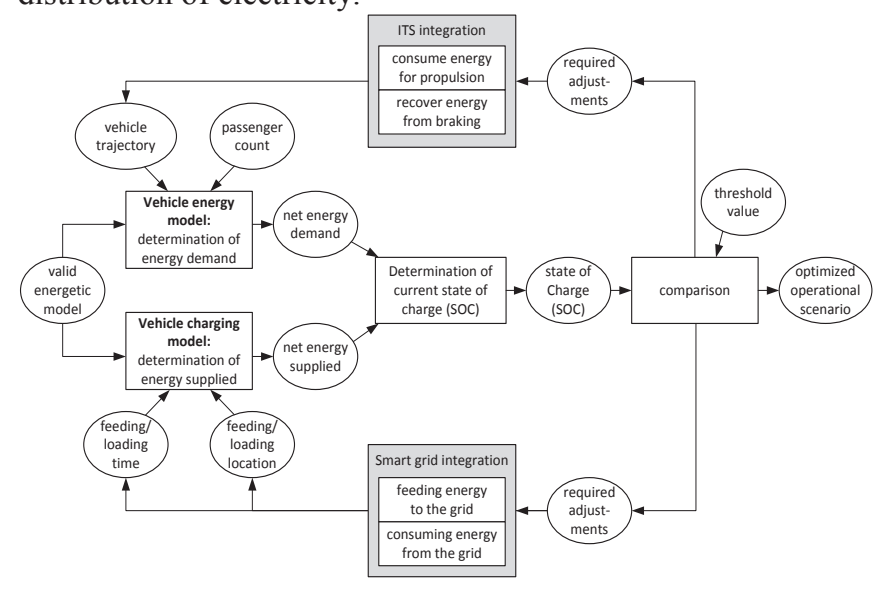

Fig. 1. Holistic approach of energetic optimization

\section{A. Energy Consumption Model}

The change of one vehicle's energy content can be calculated by summing up its kinetic, potential, and rotational energy gain components from one discrete time step to the following and subtracting the losses caused by different resistance components [5]. The vehicle's energy $E_{\mathrm{veh}}[k]$ at the discrete time step $k$ can thus be calculated by equation 1 , with the known variables vehicle mass $m$, time variant vehicle speed $v[k]$, gravity acceleration $g$, time variant vehicle altitude $h[k]$, and moment of inertia of internal rotating elements $J_{\text {int }}$.

$$
\begin{aligned}
& E_{\mathrm{veh}}[k]=E_{\mathrm{kin}}[k]+E_{\mathrm{pot}}[k]+E_{\mathrm{rot}, \mathrm{int}}[k] \\
& =\frac{m}{2} \cdot v^{2}[k]+m \cdot g \cdot h[k]+\frac{J_{\text {int }}}{2} \cdot v^{2}[k]
\end{aligned}
$$

In consideration of energy losses $\Delta E_{\text {loss }}[k]$ caused by air, rolling, and curve resistance and constant consumers (e.g. air conditioning), the energy gain between time steps $k$ and $k+1$ can be calculated by equation 2 .

$$
\Delta E_{\text {gain }}[k]=E_{\mathrm{veh}}[k+1]-E_{\mathrm{veh}}[k]-\Delta E_{\text {loss }}[k]
$$

The energy loss is made up of the components in equation 3, with the variables air density $\rho_{\text {air }}$, vehicle front surface area $A_{\text {veh}}$, air drag coefficient $c_{\mathrm{w}}$, covered distance $s[k]$, rolling resistance coefficient $c_{\text {roll }}$, centripetal force $F_{\text {rad, }}$ curve resistance coefficient $c_{\text {rad }}$, and the (average) power of constant consumers $P_{\text {const }}[9]$. 
2015 Models and Technologies for Intelligent Transportation Systems (MT-ITS) 3-5. June 2015. Budapest, Hungary

$$
\begin{gathered}
\Delta E_{\text {loss }}[k]=\Delta E_{\text {air }}[k]+\Delta E_{\text {roll }}[k]+\Delta E_{\text {curve }}[k]+\Delta E_{\text {const }}[k] \\
\Delta E_{\text {air }}[k]=\frac{1}{2} \rho_{\text {air }} \cdot A_{\text {veh }} \cdot c_{\mathrm{w}} \cdot v^{2}[k] \cdot|\Delta s[k]| \\
\Delta E_{\text {roll }}[k]=c_{\text {roll }} \cdot m \cdot g \cdot|\Delta s[k]| \\
\Delta E_{\text {curve }}[k]=c_{\text {rad }} \cdot \frac{m \cdot v^{2}[k]}{r[k]} \cdot|\Delta s[k]| \\
\Delta E_{\text {const }}[k]=P_{\text {const }} \cdot \Delta t
\end{gathered}
$$

Depending on its sign, $\Delta E_{\text {gain }}[k]$ is the amount of energy the vehicle has consumed or regained resulting from its movement and parameters. The variation of the energy contained in the vehicle's battery can further be calculated by equations 4 and 5 by introducing constant efficiency factors for recuperation $\eta_{\text {recup }}\left(\Delta E_{\text {gain }}[k]>0\right)$ and propulsion $\eta_{\text {prop }}\left(\Delta E_{\text {gain }}[k]<0\right)$.

$$
\begin{aligned}
& E_{\text {Bat }}[k+1]=E_{\text {Bat }}[k]+\Delta E_{\text {gain }}[k] \cdot \eta_{\text {recup }} \\
& E_{\text {Bat }}[k+1]=E_{\text {Bat }}[k]+\Delta E_{\text {gain }}[k] \cdot \eta_{\text {prop }}^{-1}
\end{aligned}
$$

$\square \square \square$

\section{B. Energy Charging Model}

If a vehicle moves or stops above or within a system-specific proximity of such an infrastructure element, the energy content of its battery is charged according to equation 6, with charging power $P_{\text {chrg }}$, charging efficiency $\eta_{\text {chrg, }}$, and duration between two discrete time steps $\Delta t$.

$$
E_{\mathrm{Bat}}[k+1]=E_{\mathrm{Bat}}[k]+P_{\mathrm{chrg}} \cdot \eta_{\mathrm{chrg}} \cdot \Delta t
$$

$\square \square \square$

That charging efficiency may not only comprise the electrochemical processes in the battery, but rather the overall charging process in-between the electrical grid and the vehicle. For the project emil, that included the additional losses for the energy transfer via an magnetic field and the corresponding technical devices. Thereby, additional losses for such specific charging technologies might be compared to others in an overall cost-analysis. Energy losses are to be compensated by grater charging times (e.g. no delays through mechanical connections) leading to smaller necessary vehicle energy storages or smaller impacts on time schedules.

Following the calculations of the energy variation between two discrete time steps, the battery's energy content is limited to the user-specifiable range

$$
0 \leq E_{\text {Bat }} \leq E_{\text {Bat,max }}
$$

$\square \square \square$

Calculations of this energy model can be restricted to vehicles with $E_{\mathrm{Bat}, \max }>0$, further reducing computing times.

\section{MODEL VALIDATION}

In order to use the introduced vehicle model for subsequent system design and operational planning it needs to be valid. Validity in this respect means that calculations done with the simplistic model give results that only have a tolerable deviation from the results of a far more complex model. In the case of project emil, the technology provider Bombardier Transportation $\mathrm{GmbH}$ was the source of results of a sophisticated vehicle model developed for the correct dimensioning of components for the final electric vehicles that were to be introduced in Braunschweig. No access was granted for the sophisticated model itself. . In the process of model validation, the following steps have been performed (see Figure 2):

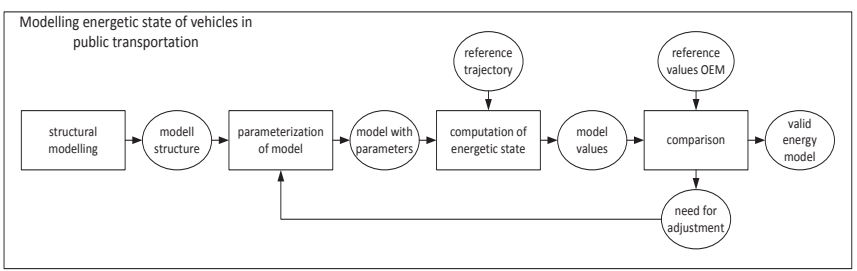

Fig. 2. Methodial approach of model validation

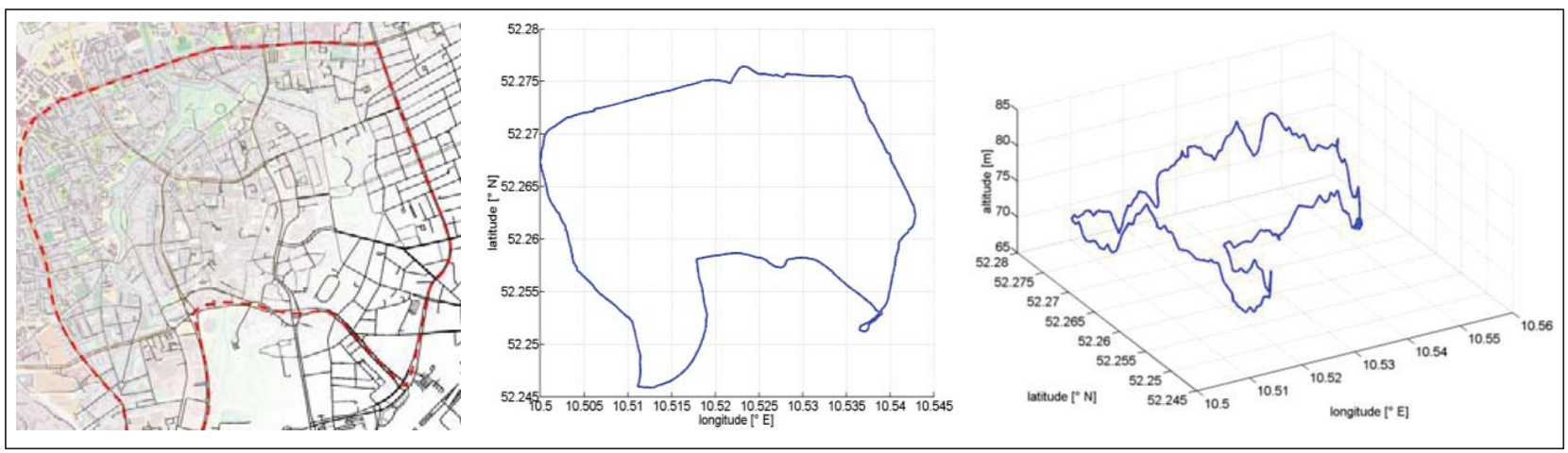

Fig. 3 Designated bus route (left, middle) and its topographic profile (bottom), COpenStreetMap-Contributors 
2015 Models and Technologies for Intelligent Transportation Systems (MT-ITS)

3-5. June 2015. Budapest, Hungary

Overall, four steps have been performed. The first step is modelling vehicle charge and discharge with the basic formulas of the simplistic model presented in the previous section. The second step is the instantiation of the simplistic model with an initial parameter set [6]. The third step is calculating the energy state of the vehicle by using the same route as the manufacturer has used for its sophisticated model and vehicle parameter set (Solaris Urbino 12). Figure 3 shows the vehicle's route and its topographic profile.

In the fourth step, the results of the simplistic model have been compared with the results of the manufacturer's sophisticated model. In multiple iterations, a parameter set of the simplistic model could be identified which represents the reference

behaviour optimally in the sense of least-squares. The reference (blue) and parameterized (red) simulation outputs, for the same route as the model input, are shown in Figure 4.

The cumulated $(\Delta t=1 \mathrm{~s})$ deviation of the two simulation outputs add up to $E_{\text {Error }}=3.3998 \mathrm{kWh}^{2}$.

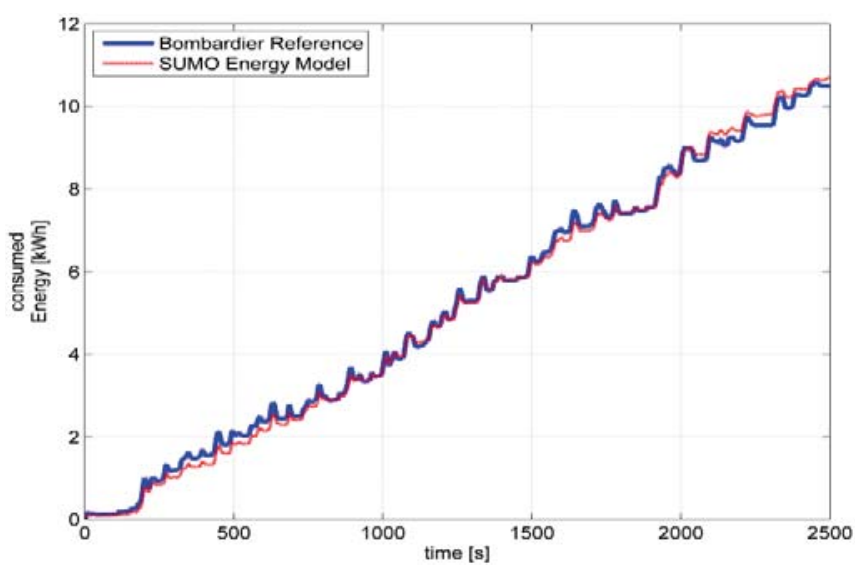

Fig. 4. Simulation outputs of Bombardier's reference simulation and the newly implemented energy model with an optimal parameter set [6]

\section{ACQUISITION AND EVALUATION OF EMPIRICAL DATA}

There are several parameters that affect the energy consumption of a vehicle as well as the charging regime that change throughout its operation. Huge variations in weather and climate can have an impact on road conditions and winds that affect rolling- and air resistance and also power consumptions of vehicle aggregates such as air conditioning needed for adequate passenger comfort. In cities on the other hand, variations in passenger rates affect dwell times at the stops. Passenger occupancy also affects the vehicle mass and therefore the overall energy consumptions. The same applies to the change of the drive cycles. Heavy traffic adds additional stops and velocity reductions at traffic lights and congestions. Induced delays due to higher dwell times and higher traffic density might partially be compensated by increased acceleration, deceleration and cruising speeds, which add up to the already increased power consumption. The charging regime on the other hand is affected by differences in stopping times. In times of heavy traffic and higher passenger dwelling times, the duration of stops and therefore the possible time of charging on the route increase, while the duration at the final stops decreases. Most of all, worst case operational scenarios must be analysed to assure that increased power consumption can be compensated by adapted energy storage capacities and charging regimes.

In the framework of the project emil, such operational scenarios were analysed by measured data from a regular diesel bus deployed on the line M19. A data logger was installed in that vehicle, and finally, the following three data sources have been used:

1. Data provided over the Fleet Management System Interface (FMS) using the network protocol SEA J1939, which allows manufacturer independent recording of relevant variables in supported commercial vehicles [7]. This interface provides information about position, basic drive train data, and data relevant for fleet management.

2. Data provided by the on-board information system (IBIS). This data includes position information (station names, geo-position and the served line ([8], for a corresponding International approach see: [9], [10]).

3. Data received from a passenger counting system based on evaluations (passenger counts) of a stereo-camera [11].

In order to define the worst case scenario for energetic optimization, the following steps have been performed:

Step 1: Identification of the time-of-day relevant for worstcase dimensioning. Figure 5 shows that in case of the M19's line section between the central station and the bus stop Madamenweg, the time slot W4 (business day, 6pm until end of business day) is the worst case to be considered for dimensioning. For the second line segment it is the time slot W1 (business day, start of operations until 9am).

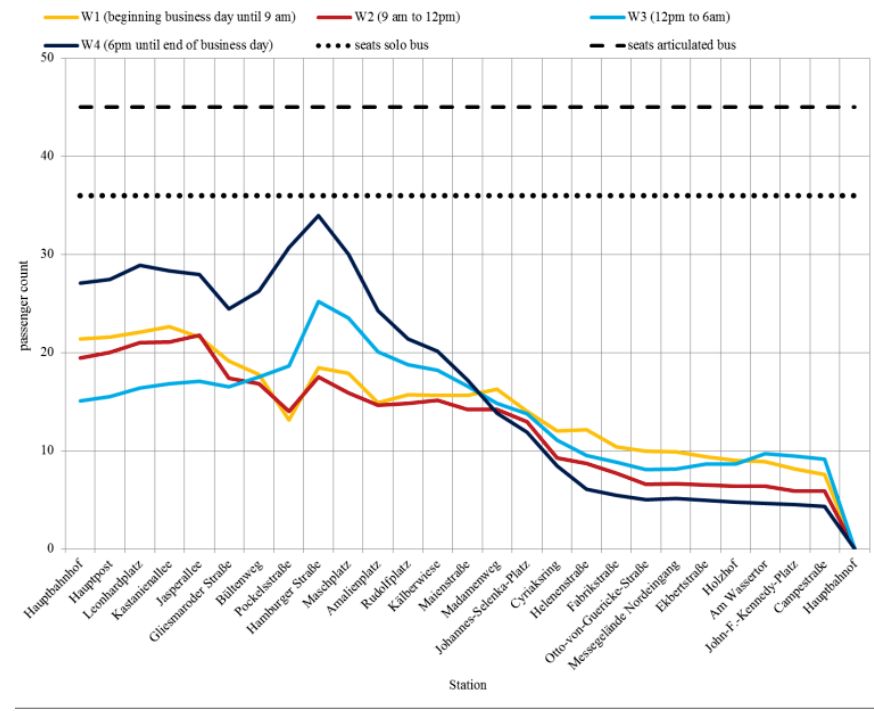

Fig. 5. average passenger occupancy at different times of day along the M19 
2015 Models and Technologies for Intelligent Transportation Systems (MT-ITS) 3-5. June 2015. Budapest, Hungary

Step 2: Identification of the minimum, average and maximum occupation along the line. The worst-case considerations from the previous step were analysed in more detail. For the adequate dimensioning, three major cases were considered: minimum passenger count, average passenger count and maximum passenger count. This allows a sensitivity analysis of battery dimensioning with respect to the parameter "passenger occupancy" (see Figure 6).

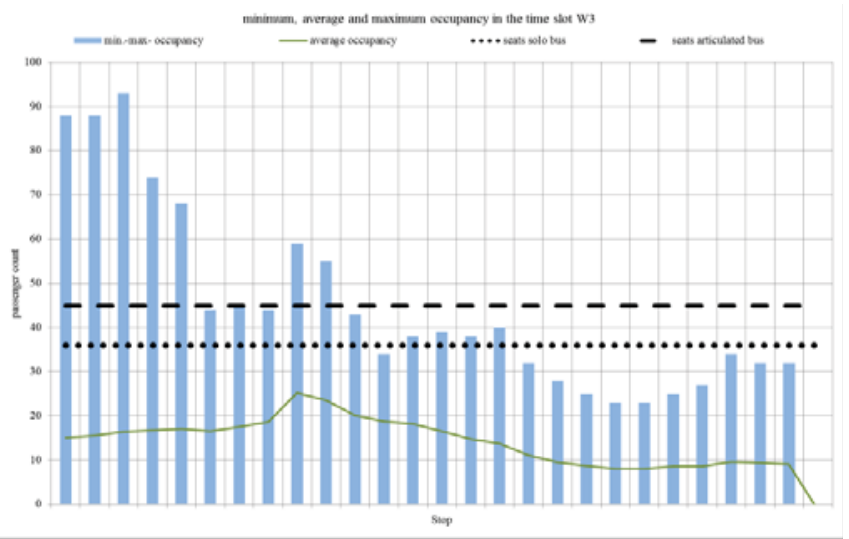

Fig. 6. minimum, average and maximum occupancy in the time slot W3

Step 3: Determination of station dwell times. In order to install the charging infrastructure on suitable stations, the station dwell times needs to be considered. The station dwell times have therefore been derived from FMS-data as well as data from the passenger counting system. These values have been double-checked by manually performed samples conducted at the same day. The results of both measurements had only small deviations.

\section{CAlCUlating ENERgy CONSUMPTION USING EMPIRICAL DATA}

After calibration, the energy model can be used to analyse measured velocity profiles, where vehicles serviced the M19 bus line. Figure 7 shows the energy consumed in the evaluated measurements. The left axis is the scale for the box plots, displaying the amount of energy consumed on each of the 26 individual segments between the 27 bus stops along the route. The right axis is the scale for the continuous plot of the average cumulated energy (per segment) over the entire route.

In both plots, the green line represents the amount of energy consumed on the individual segments with the average amount of measured passengers. The intermediate lower and upper bounds (orange in the box plot, yellow in the continuous plot) show the energy consumed on the individual segment with the minimum and maximum amount of measured passengers. The outermost bounds (red in both plots) show the energy consumed on the individual segment assuming an empty (lower) or fully occupied (upper) bus. Using the available data, the assumption for the worst-case in regard of energy consumption for an M19 trip can be corrected to $E_{\text {worstC }}$ $=22.9 \mathrm{kWh}$. It is to be emphasized that this value is nearly twice as high as the estimated average consumption. Even in regular, measured operations, the variation already reaches about $20 \%$. Such variations need to be taken into account when deciding on the energy capacity that is to be installed in the vehicles. This includes over-dimensioning which can be economic as battery life expectancy is immensely affected by great depths of discharge. That becomes clear if one considers that hybrid vehicles only use few percent of their installed energy capacity to allow a range of several hundred thousand charging and discharging cycles over a lifetime of more than ten years [12].

After having identified the energy consumptions, the possibilities for charging need to be evaluated. Due to the fact that a public transportation system is regarded, vehicle stops will occur at predefined locations. These locations shall be evaluated for their suitability for intermediate vehicle charging.

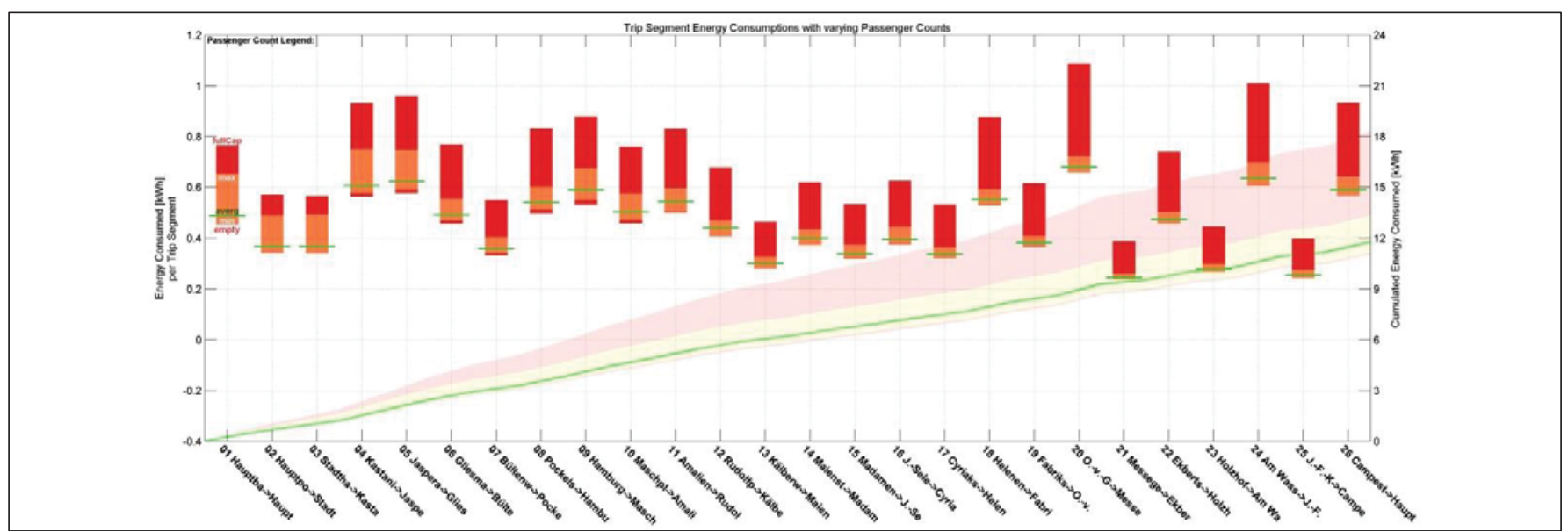

Fig. 7. Energy consumption along the M19 route for individual segments (box plots, scaled by left axis) and cumulated (continuous line, scaled by right axis) 


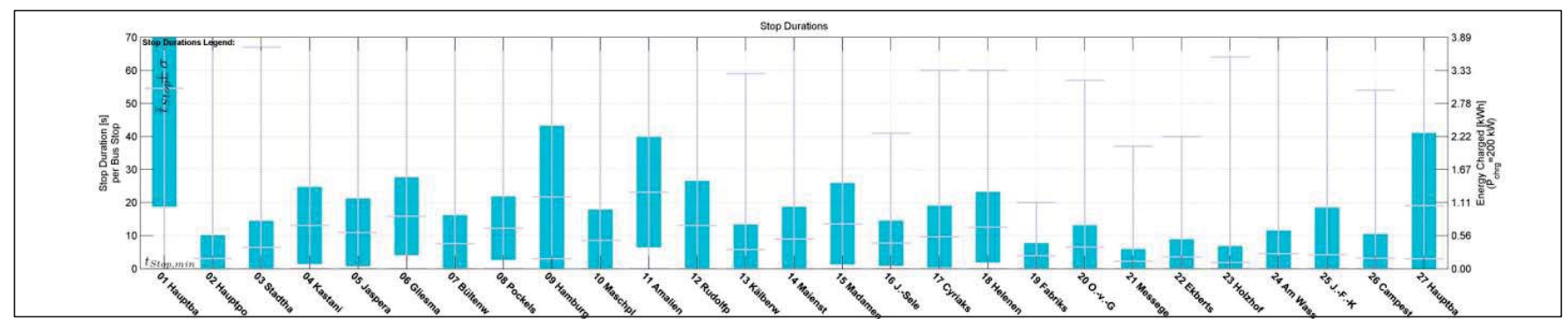

Fig. 8. Mean halt durations (left axis) and corresponding energy that could be charged within that time with a charging power Pchrg $=200 \mathrm{~kW}$ (right axis)

Among the measured FMS variables, information is provided about the door status. It is a variable whose value is zero if all doors are closed and non-zero if any door is open. This variable, along with the criterion that a vehicle needs to be in proximity of a charging station, has been used to identify vehicle halts, which are suitable for charging. Figure 8 illustrates the stopping probability and dwell time at individual stops along the route of the M19.

The cyan bars span across two standard deviations, with the mean dwell time in the centre indicated by a black line. The non-centred upper and lower black lines indicate the maximum and minimum observed halt durations at the corresponding bus stop. The left axis scales the dwell time, whereas the right axis scales the corresponding energy, that could be charged within that period and with a charging power of $P_{\text {chrg }}=200 \mathrm{~kW}$ (power of actual charging technology installed).

It is evident, that the bus stops 8 (Hamburger Straße) and 10 (Amalienplatz) would be most suitable for intermediate charging, each allowing for an average recharge of approximately $1.2 \mathrm{kWh}$. In terms of the worst-case scenario $\left(E_{\text {worstC }}=22.9 \mathrm{kWh}\right)$, this means that only $20.5 \mathrm{kWh}$ would need to be recharged at the terminal stop, reducing the required time for charging to only 6.2 minutes at a charging power of $P_{\text {chrg }}=200 \mathrm{~kW}$. If rigging each bus stop with a charging station would be economically profitable, the required energy to be recharged could further be reduced to $10.46 \mathrm{kWh}$ and the corresponding duration to only 3.1 minutes.

\section{CONCLUSION AND OUTLOOK}

First results of the project indicate that for future work, a more differentiated view on time losses of public transport vehicles during their operation is required. Currently the German Aerospace Center (DLR) is implementing a vehicle platform on board of local buses. For an in-depth analysis of time losses at intersections with traffic light controllers, transceivers for the IEEE $802.11 \mathrm{p}$ communication standard shall be integrated into the on-board platform [13]. This allows to receive data about signal phase and timing (SPAT) of traffic light controllers. Within the application platform for intelligent mobility (AIM - a large scale research infrastructure operated by DLR) 35 cooperative traffic light controllers along the line M19 are available for data analysis [14]. In addition to this, further effort has to be put on the analysis of FMS data as this allows to determine time losses due to dense traffic conditions (stop-and-go). In addition to further acquisition of empirical data, the applied models will be enhanced with the goal of improving vehicle trajectory planning.

The primary aspect of the project emil was the worldwide first introduction of inductively charged vehicles into regular line bus services. This proves that this technology is suitable for urban bus operations. Despite successful demonstration of technological aspects, economical aspects deserve more careful investigation. In this context, the already existing simulation tools would allow for an extension that can assess cost effectiveness of selected vehicles and corresponding infrastructure and their optimization. This includes levelling peaks of energy consumption by planning vehicle trajectories in a way which prevents that two vehicles use the same charging infrastructure at the same time. An approach for such an optimization was given by the project CACTUS [15].

\section{ACKNOWLEDGMENT}

The project emil is funded by the German Federal Ministry of Transport and digital infrastructure (Bundesministerium für Verkehr und digitale Infrastruktur - BMVI). We hereby thank all our project partners for their continuous and kind cooperation.

\section{REFERENCES}

[1] Bunte, Stefan: Lösungen für Anwendungsfälle der Fahrzeugeinsatzplanung im öffentlichen Personennahverkehr. Dissertation, Universität Paderborn, 2009.

[2] Büchter, H.; Naumann, S.: Hybrides Planungswerkzeug für die Infrastruktur elektrisch betriebener Busse im öffentlichen Nahverkehr. Accepted for publishing in Der Nahverkehr 5/2015.

[3] Meins, J.; Soyck, F.; Engel, B.; Kurczveil, T.; Schnieder, E.: Application of high-power inductive charging of electric buses in scheduled line service. HEV2014 - 11. Symposium Hybrid- und Elektrofahrzeuge, Braunschweig, Deutschland, Februar 2014

[4] Directive 2010/40/EU of the European Parliament and of the Council of July 2010.

[5] Mitschke, M., Wallentowitz, H. Dynamik der Kraftfahrzeuge. Springer, Berlin (2004)

[6] Kurczveil, T.; Schnieder, E.: Extending a Traffic Simulation Tool for the Evaluation of novel Charging Infrastructures. Electric Drives Production Conference (EDPC), $20133^{\text {rd }}$ International, pp. 1-5.

[7] HDEI/BCEI Working Group: Fleet Management System Standard Description, Version 03, 2012

[8] Verband Deutscher Verkehrsunternehmen (VDV): Integriertes Bordinformationssystem (IBIS), Ergänzung 2; VDV Schriften 300 7/91 
2015 Models and Technologies for Intelligent Transportation Systems (MT-ITS)

3-5. June 2015. Budapest, Hungary

[9] European Bus System of the future; web site. http://www.ebsf.eu/

[10] Sciandra, V. ; Agueh, M. ; George, L. ; Anjary, A. ; Cochet, P. ; Jomaa, M. ; Gharbi, W. ; El Merrouni Alami, D.: A smart communication gateway for V2I applications in Public Transport; Communications Conference (COLCOM), 2012 IEEE Colombian, 2012 , pp.: 1 - 6

[11] Verband Deutscher Verkehrsunternehmen (2007): VDV-Schrift 457 Rahmenlastenheft Automatische Fahrgastzählsysteme (AFZS). VDV, Köln.

[12] Thomas B. Reddy, Linden's Handbook of Batteries, McGraw-Hill, 2011
[13] Frankiewicz, Tobias; Schnieder, Lars; Köster, Frank: Application Platform for Intelligent Mobility Test Site Architecture and Vehicle2X Communication Setup. 19. ITS World Congress. Wien. 2012.

[14] Schnieder, Lars: Entwicklung intelligenter Mobilitätsdienste im realen Verkehrsumfeld in der Anwendungsplattform Intelligenten Mobilität. 13. Fachtagung Entwurf komplexer Automatisierungssysteme (EKA), Magdeburg, 14./15.05.2014.

[15] CACTUS. Models and Methods for the Evaluation and the Optimal Application of Battery Charging and Switching Technologies for Electric Busses, IFAK Magdeburg-Fraunhofer IML Stuttgart, Silesian University of Technology Katowice, Magdeburg-Stuttgart-Katowice 2012-2015 